INPLASY

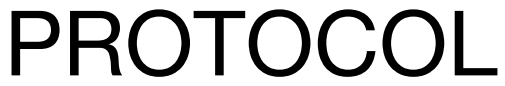

To cite: Zheng et al. Prognostic and Clinicopathological Importance of MicroRNA-140 Expression in Cancer Patients. Inplasy protocol 202180037. doi:

10.37766/inplasy2021.8.0037

Received: 10 August 2021

Published: 10 August 2021

Corresponding author: Jianhua Liao

ljhsywzh@163.com

Author Affiliation:

Zhejiang Hospital.

Support: No.

Review Stage at time of this submission: Data extraction.

Conflicts of interest:

None declared.

\section{Prognostic and Clinicopathological Importance of MicroRNA-140 Expression in Cancer Patients}

Review question / Objective: MicroRNA-140 (miR-140) is one of the most widely investigated miRNAs in cell carcinogenesis and cancer development. Despite present proposals of employing miR-140 as a candidate biomarker for cancer prognosis, its effectiveness in predicting patient survival and clinicopathological outcome is still under debate. Thus, we specifically performed this meta-analysis to examine the prognostic ability of miR-140 levels in cancer.

Information sources: We carefully performed an exhaustive search for English publications using online databases, like PubMed, Embase and the Cochrane Library from the inception of the databases to May 10, 2021. Additionally, to conduct a thorough search of all relevant papers, we also scanned the references of all eligible papers to find publications that were missed in the previous search. Two authors separately reviewed all articles.

INPLASY registration number: This protocol was registered with the International Platform of Registered Systematic Review and Meta-Analysis Protocols (INPLASY) on 10 August 2021 and was last updated on 10 August 2021 (registration number INPLASY202180037).

\section{INTRODUCTION}

Review question / Objective: MicroRNA-140 (miR-140) is one of the most widely investigated miRNAs in cell carcinogenesis and cancer development. Despite present proposals of employing miR-140 as a candidate biomarker for cancer prognosis, its effectiveness in predicting patient survival and clinicopathological outcome is still under debate. Thus, we specifically performed this meta-analysis to examine the prognostic ability of miR-140 levels in cancer.

Rationale: We explored the association between miR-140 levels and 
clinicopathological features (CPF) and patient prognosis in cancer by conducting an exhaustive review of relevant literature, with a goal to clarify the role of miR-140 in cancer.

Condition being studied: MicroRNA-140 (miR-140) is one of the most widely investigated miRNAs in cell carcinogenesis and cancer development. Despite present proposals of employing $\mathrm{miR}-140$ as a candidate biomarker for cancer prognosis, its effectiveness in predicting patient survival and clinicopathological outcome is still under debate. Here, we explored the association between miR-140 levels and clinicopathological features and patient prognosis in cancer by conducting an exhaustive review of relevant literature, with a goal to clarify the role of miR-140 in cancer.

\section{METHODS}

Search strategy: We carefully performed an exhaustive search for English publications using online databases, like PubMed, Embase and the Cochrane Library from the inception of the databases to May 10, 2021. We employed keywords like "microRNA-140 OR miR-140" AND "cancer or tumor or malignancy or neoplasm or carcinoma" AND "prognosis or prognostic or survival or outcome".

Participant or population: Adult participants received a pathological diagnosis of cancer and received reasonable and effective therapeutic measures will be included.

Intervention: All participants were separated into cohorts, based on their miR-140 levels, and survival analysis was completed on both cohorts.

Comparator: We will analyze clinicopathologic and survival differences in cancer patients with different miR-140 expressions.

Study designs to be included: Randomized controlled trials will be included.
Eligibility criteria: The following articles were including in our analysis: (1) all adult participants received a pathological diagnosis of cancer and received reasonable and effective therapeutic measures; (2) examination of miR-140 levels in cancerous tissues or blood; (3) all participants were separated into cohorts, based on their miR-140 levels, and survival analysis was completed on both cohorts; (4) sufficient data were provided to measure the hazard ratio (HR) and 95\% confidence intervals $(\mathrm{Cl})$; and (5) studies involved more than 50 enrolled patients. Any articles that failed to comply with the above criteria were eliminated from our analysis. In addition, case reports, reviews, conference abstracts, letters and animal trials were also excluded.

Information sources: We carefully performed an exhaustive search for English publications using online databases, like PubMed, Embase and the Cochrane Library from the inception of the databases to May 10, 2021. Additionally, to conduct a thorough search of all relevant papers, we also scanned the references of all eligible papers to find publications that were missed in the previous search. Two authors separately reviewed all articles.

Main outcome(s): Our meta-analysis, which synthesized the results of all eligible studies, showed that expression of miR-140 was associated with survival and clinicopathological features in cancer patients. Therefore, we have reason to believe that miR-140 is not only a standalone indicator of patient survival but may also become a new target for cancer therapy.

Additional outcome(s): Based on the current research focus on miRNAs, this we aimed to find a therapeutic direction to further improve the prognosis of tumor patients.

Data management: All data was separately compiled by two scientists. Relevant data included authors, publication year, research location, recruitment duration, population size, cancer type, detection 
procedure, detected sample, threshold, analysis type, HR prediction, and clinicopathological features.

Quality assessment / Risk of bias analysis: Study quality was assessed according to the Newcastle-Ottawa Quality Assessment Scale (NOS). Begg's and Egger's tests analyzed publication bias.

Strategy of data synthesis: HRs and their subsequent $95 \% \mathrm{Cls}$ were pooled to examine the association between miR-140 levels and the overall survival (OS) of cancer patients, and HRs from multivariate analyses in each study were preferentially included in the analysis. The relationship between miR-140 levels and CPF was examined by pooling the odds ratios (ORs) and their subsequent $95 \% \mathrm{Cls}$. The chisquare test and 12 statistic were employed to test heterogeneity. A fixed-effects model was used if the $P$ value exceeded 0.05 and/ or the 12 was less than $50 \%$. Otherwise, the random-effects model was employed.

Subgroup analysis: Subgroup analyses were performed according to therapy method, cancer type, analysis method, publication date, sample size, cutoff value, detected sample and clinicopathological features.

Sensitivity analysis: Sensitivity analysis was carried out by sequentially omitting individual study to validate the stability of the pooled outcomes based on the overall HR estimate of OS.

Language: English.

Country(ies) involved: China.

Keywords: MicroRNA-140, Cancer, Prognosis.

Contributions of each author:

Author 1 - Mengxia Zheng.

Author 2 - Jingting Liu.

Author 3 - Chunyan Meng.

Author 4 - Kaifeng Tang.

Author 5 - Jianhua Liao. 